Submitted to ApJ Letters on 2013 September 10

Preprint typeset using LTEX style emulateapj v. 04/17/13

\title{
REVIVAL OF THE STALLED CORE-COLLAPSE SUPERNOVA SHOCK TRIGGERED BY PRECOLLAPSE ASPHERICITY IN THE PROGENITOR STAR
}

\author{
SEAN M. COUCH ${ }^{1, *}$ AND Christian D. OTT ${ }^{2,3,+}$ \\ Submitted to ApJ Letters on 2013 September 10
}

\begin{abstract}
Multi-dimensional simulations of advanced nuclear burning stages of massive stars suggest that the Si/O layers of presupernova stars harbor large deviations from the spherical symmetry typically assumed for presupernova stellar structure. We carry out three-dimensional core-collapse supernova simulations with and without aspherical velocity perturbations to assess their potential impact on the supernova hydrodynamics in the stalled shock phase. Our results show that realistic perturbations can qualitatively alter the postbounce evolution, triggering an explosion in a model that fails to explode without them. This finding underlines the need for a multi-dimensional treatment of the presupernova stage of stellar evolution.
\end{abstract}

Keywords: hydrodynamics - neutrinos - Stars: supernovae: general

\section{INTRODUCTION}

The core-collapse supernova (CCSN) phenomenon is fundamentally multi-dimensional. Axisymmetric (2D) and threedimensional (3D) simulations have shown that convection and the standing accretion shock instability (SASI) robustly break spherical symmetry in the pre-explosion stalled-shock phase (see, e.g., Couch \& O'Connor 2013; Couch 2013; Ott et al. 2013; Dolence et al. 2013; Hanke et al. 2013; Takiwaki et al. 2013 for recent 3D simulations). The propagation of artificially initiated explosions through the progenitor envelope found that symmetry is broken by Rayleigh-Taylor and Richtmyer-Meshkov instabilities (e.g., Couch et al. 2009; Hammer et al. 2010; Joggerst et al. 2010). The conclusions of these simulations are backed up by observations of asphericities in local supernova remnants (Vink 2012), by spectropolarimetry of distant CCSNe (Wang \& Wheeler 2008; Chornock et al. 2011, and references therein), and by pulsar kicks (e.g., Hobbs et al. 2005).

For initial conditions based on 1D stellar evolutionary models, the breaking of spherical symmetry after the initial collapse and bounce of the inner core is widely appreciated. Stars, however, are not truly spherical. Yet, the current stateof-the-art in CCSN progenitor evolution is 1D. Such models resort to various kludges to account for multi-D phenomena such as convection, rotation, and magnetic fields (see Langer 2012 for a review). Exploratory explicit multi-D hydrodynamics simulations of the $\mathrm{Si} / \mathrm{O}$-shell burning stage prior to core collapse (Bazan \& Arnett 1998; Meakin \& Arnett 2007; Arnett \& Meakin 2011) have shown that violent fluctuations about the mean turbulent flow can lead to low-mode deviations from spherical symmetry. These fluctuations may also trigger eruptions that partially unbind the stellar envelope, leading to precursor transients weeks to months prior to core collapse (Smith \& Arnett 2013, but also see Quataert \& Sh-

\footnotetext{
${ }^{1}$ Flash Center for Computational Science, Department of Astronomy \& Astrophysics, University of Chicago, Chicago, IL, 60637, smc@flash.uchichago.edu

2 TAPIR, Mailcode 350-17, California Institute of Technology, Pasadena, CA 91125, USA, cott@tapir.caltech.edu

${ }^{3}$ Kavli Institute for the Physics and Mathematics of the Universe (Kavli IPMU WPI), The University of Tokyo, Kashiwa, Japan

* Hubble Fellow

+ Alfred P. Sloan Research Fellow
}

iode 2012). This has now been observed for multiple CCSNe. The fluctuations and their consequences cannot be captured by the standard mixing-length approach for convection and time-implicit stellar evolution codes (Smith \& Arnett 2013).

The perturbations caused by Si/O shell burning fluctuations are part of the supersonically collapsing outer core and may be amplified during collapse (Lai \& Goldreich 2000). They reach the stalled shock $\sim 100-300 \mathrm{~ms}$ after bounce, depending on the structure of the progenitor. At this time, neutrinodriven convection and/or SASI are active and may be affected by spatial variations in the accretion flow. Burrows \& Hayes (1996) were the first to carry out 2D collapse simulations of a progenitor whose density outside $0.9 M_{\odot}$ was decreased by $15 \%$ within a $20^{\circ}$ wedge of the pole. They found an early explosion in the direction of the perturbation and a hydrodynamically kicked protoneutron star. Fryer (2004), studied similarly large $\ell=1$ perturbations applied globally, or only in the Si/O layers, using 3D smooth particle hydrodynamics. $\mathrm{He}$ also found neutron star kicks and explosion asymmetries, though of smaller magnitude than observed in 2D.

In this Letter, we examine the role of perturbations on the explosion mechanism itself. We carry out 3D simulations of the postbounce evolution of a nonrotating $15-M_{\odot}$ progenitor star. Unlike previous work, we apply momentum-preserving tangential velocity perturbations with spatial frequency and magnitude motivated by Bazan \& Arnett (1998) and Arnett \& Meakin (2011). We also carry out unperturbed control simulations for comparison. Our results demonstrate that asphericities in the $\mathrm{Si} / \mathrm{O}$ layer increase the strength of turbulence behind the stalled but dynamic shock. This creates conditions more favorable for shock expansion. We show that the perturbations can trigger explosion in a model that would not explode otherwise.

\section{METHODS AND SETUP}

We simulate 3D Newtonian CCSN postbounce evolution using the FLASH simulation framework (Fryxell et al. 2000; Dubey et al. 2009; Lee et al. 2013). ${ }^{6}$ Our basic numerical approach is described by Couch \& O'Connor (2013) and Couch (2013). We use the multispecies neutrino leakage scheme of O'Connor \& Ott (2010), whose 3D version was also employed in Ott et al. (2012); Ott et al. (2013). The neutrino

${ }^{6}$ Available at http://flash.uchicago.edu. 


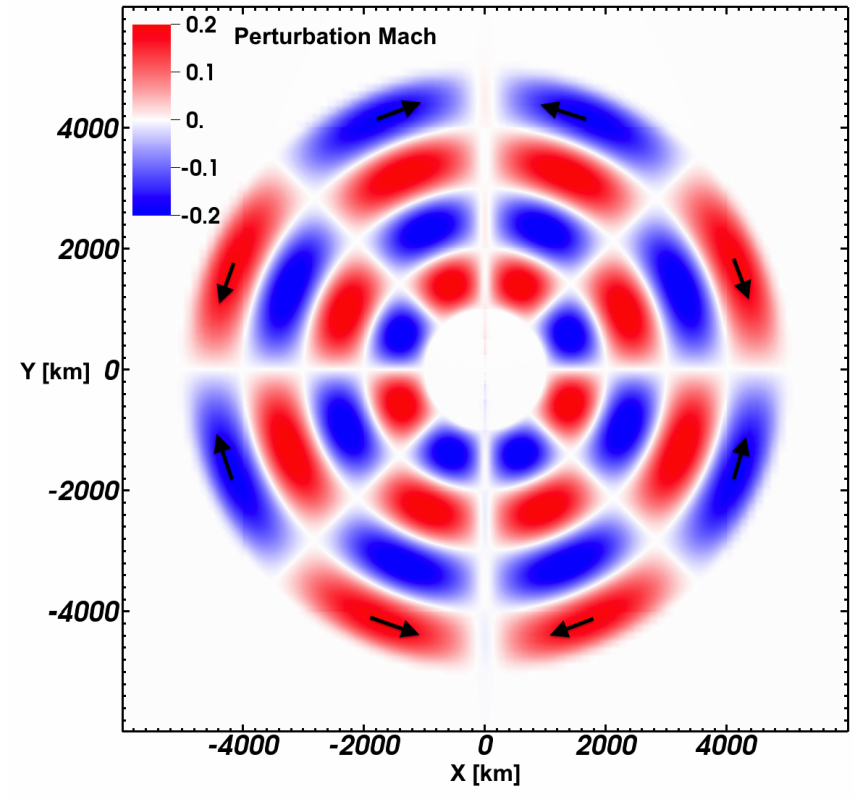

Figure 1. Example of the initial $\theta$-velocity perturbations applied in this study. Shown is the a meridional slice of the Mach number of the $\theta$-direction velocity. The arrows in the outer ring of perturbations show the local velocity directions.

leakage scheme includes a multiplicative factor, $f_{\text {heat }}$, in the neutrino heating source term, which can be adjusted to yield more efficient neutrino heating (i.e., $f_{\text {heat }}>1$ ). The leakage scheme with $f_{\text {heat }}=1.00$ is tuned to match the multiangle, multigroup full neutrino transport simulations of Ott et al. (2008). In all simulations reported here, we use 3D Cartesian geometry with a finest grid spacing $d x_{\min }=0.49 \mathrm{~km}$. Using adaptive mesh refinement, we achieve a pseudo-logarithmic grid by decrementing the maximum allowed refinement level as a function of radius. The typical effective "angular" resolution is $0.37^{\circ}$.

We use a single progenitor model, the $15-M_{\odot}$ star of Woosley \& Heger (2007). In order to study the dependence of 3D CCSN simulations on asphericities extant in the progenitor, we apply perturbations to the 1D stellar profile. We seed perturbations that are convolutions of sinusoidal functions of radius and angle. For simplicity, we perturb only the velocity in the spherical $\theta$-direction and leave all other variables untouched. The form of the sinusoidal perturbation to $v_{\theta}$ is

$$
\delta v_{\theta}=M_{\text {pert }} c_{S} \sin [(n-1) \theta] \sin [(n-1) \zeta] \cos (n \phi),
$$

where $M_{\text {pert }}$ is the peak Mach number of the perturbations, $c_{S}$ is the local adiabatic sound speed, $n$ is the number of nodes in the interval $\theta=[0, \pi]$, and $\zeta=\pi\left(r-r_{\text {pert,min }}\right) /\left(r_{\text {pert,max }}-\right.$ $\left.r_{\text {pert,min }}\right)$. The perturbations are only applied within a spherical shell with radial limits $r_{\text {pert,min }}<r<r_{\text {pert,max }}$. We scale the perturbations with local sound speed so that the peak amplitudes of the perturbations are constant in Mach number, not absolute velocity. This results in higher-speed perturbations at smaller radii where the sound speeds are larger. Importantly, for odd node numbers, Eq. (1) results in zero net momentum contribution to the inital conditions. We have verified this experimentally to machine-precision.

\section{RESULTS}

We start our 3D simulations from the results of 1D simulations at $2 \mathrm{~ms}$ after core bounce, and it is at this point that we apply the perturbations given by Eq. (1). In the results we discuss here, we use a node count $n=5$ and peak perturbation Mach number $M_{\text {pert }}=0.2$. This establishes large-scale perturbations that are similar in extent and speed to some convective plumes found in multi-D progenitor burning simulations (Meakin \& Arnett 2007; Arnett \& Meakin 2011). We choose $r_{\text {pert,min }}$ to correspond to the inner edge of the silicon shell (i.e., the outer edge of the iron core). For this progenitor at the time of core bounce, this corresponds to a radius of $\sim 1000$ $\mathrm{km}$. We set $r_{\text {pert,max }}=5000 \mathrm{~km}$, which is sufficiently large to never reach the shock during the simulated time period. Figure 1 shows a pseudo-color plot of the perturbations used in this study.

We present the results of four 3D simulations, two perturbed and two unperturbed. We use two different heat factors for both perturbed and unperturbed case: $f_{\text {heat }}=1.00$ and a slightly enhanced heating case with $f_{\text {heat }}=1.02$. We refer to the simulations using the scheme $\mathrm{n}$ [node count] $\mathrm{m}$ [initial perturbation Mach number, times ten] $f_{\text {heat }}$ [heat factor], such that the perturbed model with enhanced heat factor is referred to as ' $\mathrm{n} 5 \mathrm{~m} 2 \mathrm{f}_{\text {heat }} 1.02$.'

We find that introducing plausibly-scaled velocity perturbations in the $\mathrm{Si}$ shell of the progenitor star can trigger a successful explosion for cases in which an unperturbed simulation fails. Figure 2 shows several entropy volume renderings for models $\mathrm{n} 0 \mathrm{~m} 0 f_{\text {heat }} 1.02$ and $\mathrm{n} 5 \mathrm{~m} 2 f_{\text {heat }} 1.02$ at three postbounce times. The only difference between these two models is the presence of initial velocity perturbations in the $\mathrm{Si} / \mathrm{O}$ layer. Model n5m2 $f_{\text {heat }} 1.02$ results in continued runaway shock expansion and asymmetric explosion, as clearly shown, while model n0m0 $f_{\text {heat }} 1.02$ fails to explode and the shock recedes to small radii. At $100 \mathrm{~ms}$, only shortly after the perturbations have reached the shock, both simulations are quite similar showing strong convection following the preceding period of shock expansion. By $200 \mathrm{~ms}$, however, differences in the models are obvious. The shock has already begun to recede in $\mathrm{n} 0 \mathrm{~m} 0 f_{\text {heat }} 1.02$ while model $\mathrm{n} 5 \mathrm{~m} 2 f_{\text {heat }} 1.02$ has retained a large shock radius and is on the verge of runaway shock expansion. The last frames show the final states of the two simulations. Model n5m $2 f_{\text {heat }} 1.02$ has exploded, resulting in a large, asymmetric shock structure, while the shock has fallen back to $\sim 100 \mathrm{~km}$ in model n0m0 $f_{\text {heat }} 1.02$.

In Figure 3, we present the time evolutions of several global metrics for our four 3D simulations. The top panel of Fig. 3 shows the average shock radius. All models, with the exception of $\mathrm{n} 5 \mathrm{~m} 2 f_{\text {heat }} 1.02$, fail to explode. Compared with the control case, $\mathrm{n} 0 \mathrm{~m} 0 f_{\text {heat }} 1.00$, both $\mathrm{n} 0 \mathrm{~m} 0 f_{\text {heat }} 1.02$ and $\mathrm{n} 5 \mathrm{~m} 2$ $f_{\text {heat }} 1.00$ show longer stalled-shock phases prior to shock recession. These two intermediate cases, despite employing different heat factors, show remarkably similar average shock radius histories. In the case of the successful explosion, n5m2 $f_{\text {heat }} 1.02$, the average shock radius remains extremely similar to the comparable unperturbed model, n0m0 $f_{\text {heat }} 1.02$, until about $100 \mathrm{~ms}$ after bounce. The average shock radius of $\mathrm{n} 5 \mathrm{~m} 2 f_{\text {heat }} 1.02$ remains relatively constant just below $200 \mathrm{~km}$ until $t_{\mathrm{pb}} \sim 200 \mathrm{~ms}$ at which point the shock begins to expand rapidly, signaling the onset of explosion.

The second panel of Fig. 3 shows a measure of the overall shock asymmetry, the normalized standard deviation of the shock radius $\tilde{\sigma}$. The shock asymmetry grows as $n 5 \mathrm{~m} 2$ $f_{\text {heat }} 1.02$ experiences runaway shock expansion, indicating that the explosion is aspherical, as is also clear from the bottom-right panel of Fig. 2. The failed explosions show comparatively small values of $\tilde{\sigma}$, implying relative sphericity of 

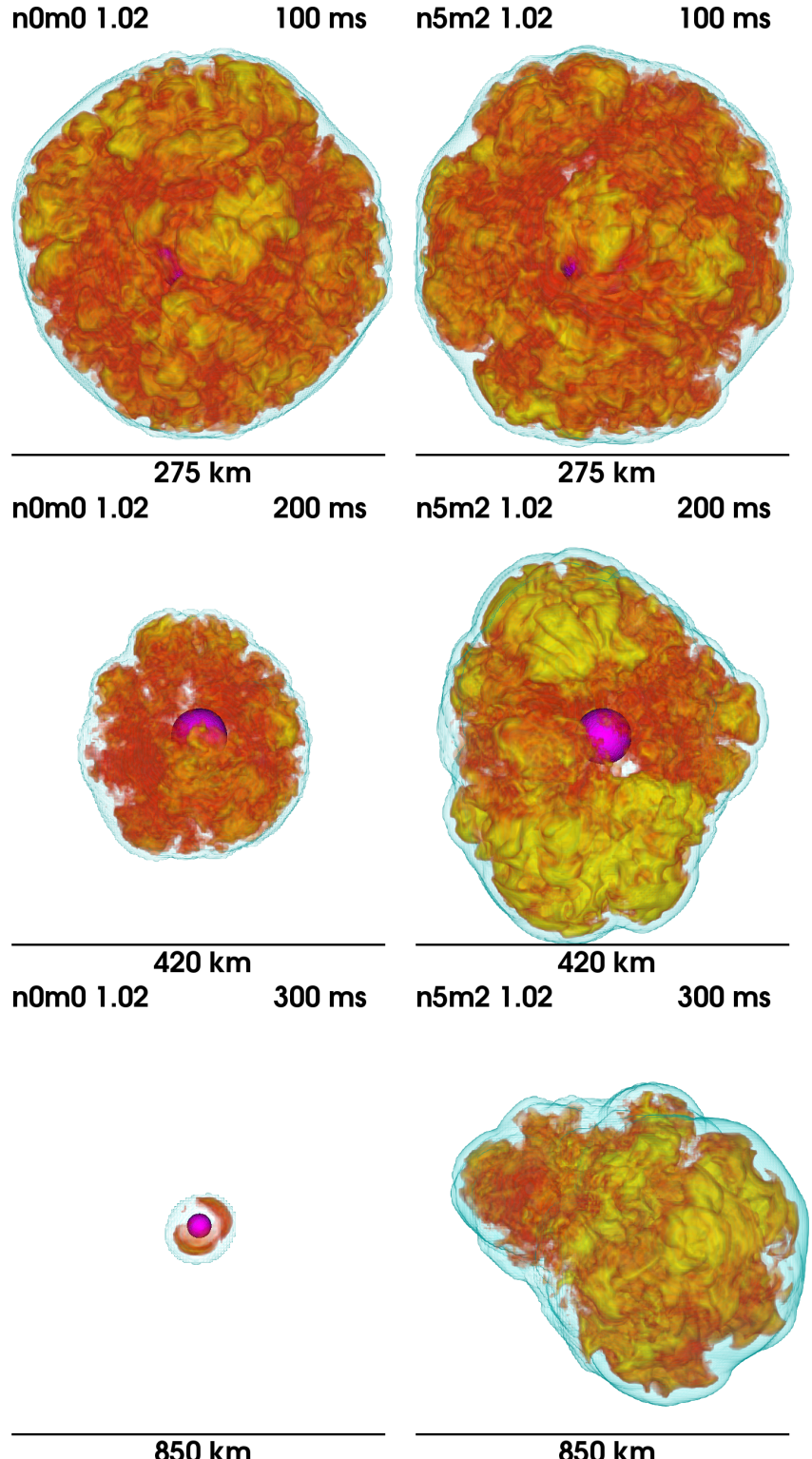

Figure 2. Volume renderings of entropy for models n $0 \mathrm{~m} 0 f_{\text {heat }} 1.02$ (left column) and $\mathrm{n} 5 \mathrm{~m} 2 f_{\text {heat }} 1.02$ (right column) at three different postbounce times, from top to bottom: $100 \mathrm{~ms}, 200 \mathrm{~ms}$, and $300 \mathrm{~ms}$. The spatial scale is noted at the bottom of each pane and increases with time. The PNS is visible in the center of the renderings, marked by a magenta constant-density contour with value $10^{12} \mathrm{~g} \mathrm{~cm}^{-3}$.

the shock surface, until strong SASI oscillations set in after the shock has receded (see Couch \& O'Connor 2013).

The presence of pre-shock perturbations has substantial impact on the neutrino heating efficiency, $\eta=Q_{\text {net }}\left(L_{\nu_{e}}+\right.$ $\left.L_{\overline{\nu_{e}}}\right)^{-1}$. As shown in the third panel of Fig. 3, for $n 5 \mathrm{~m} 2$ $f_{\text {heat }} 1.00$, the heating efficiency history is very similar to that of n $0 \mathrm{~m} 0 f_{\text {heat }} 1.02$. This implies that the perturbations drive nonradial motion that increases the dwell time of material in the gain region, significantly enhancing the fraction of neutrino luminosity absorbed. For $\mathrm{n} 5 \mathrm{~m} 2 f_{\text {heat }} 1.02$, the combination of $f_{\text {heat }}>1$ and pre-shock perturbations results in a sufficiently increased heating efficiency to initiate a neutrinodriven explosion. Also, $\eta$ depends sensitively, and nonlinearly, on $f_{\text {heat }}$. The time-averaged heating efficiencies for simulations $\mathrm{n} 0 \mathrm{~m} 0 f_{\text {heat }} 1.00, \mathrm{n} 0 \mathrm{~m} 0 f_{\text {heat }} 1.02, \mathrm{n} 5 \mathrm{~m} 2 f_{\text {heat }} 1.00$,

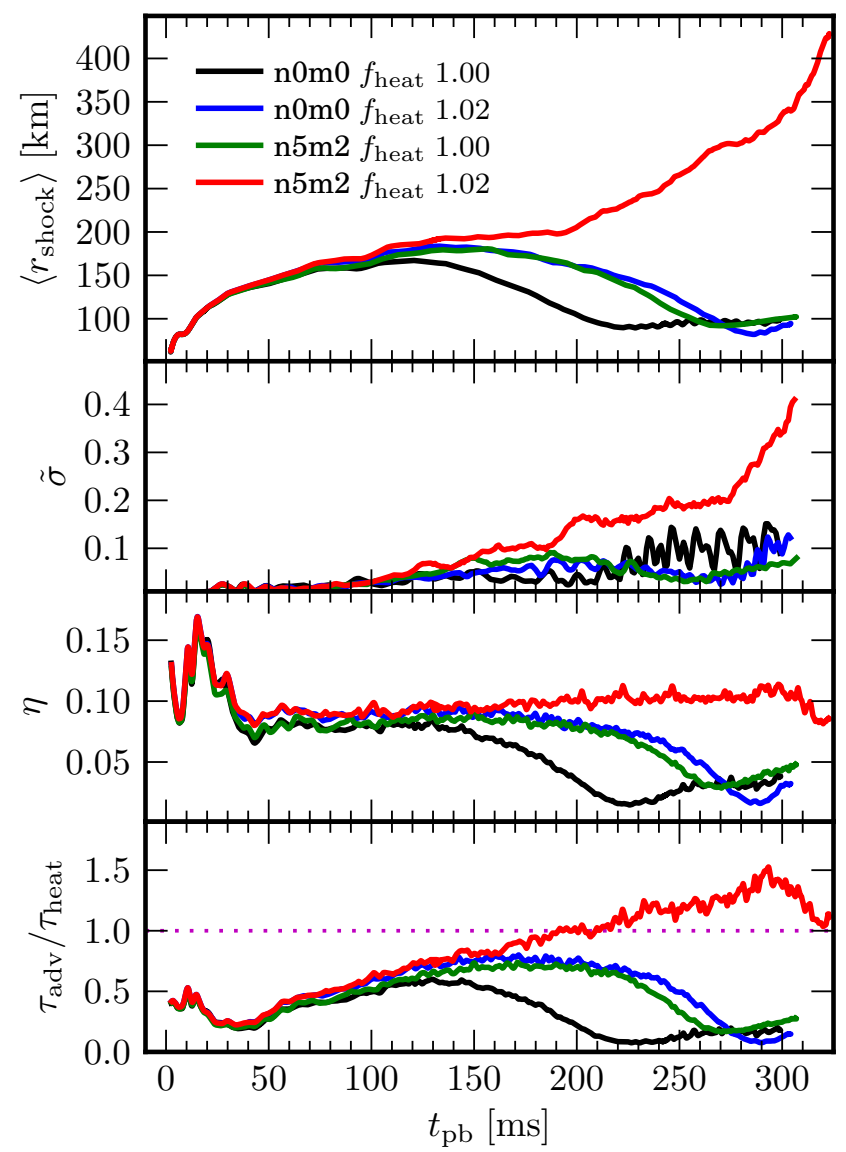

Figure 3. Time evolution of the global explosion diagnostics for our simulations. Four 3D simulations are shown: unperturbed models with $f_{\text {heat }} 1.00$ (black lines) and 1.02 (blue lines), and perturbed models with $f_{\text {heat }} 1.00$ (green lines) and 1.02 (red lines). The top panel shows the average shock radius. The second panel shows the normalized standard deviation of the shock radius, $\tilde{\sigma}=\left\langle r_{\text {shock }}\right\rangle^{-1}\left[(4 \pi)^{-1} \int d \Omega\left(r_{\text {shock }}-\left\langle r_{\text {shock }}\right\rangle\right)^{2}\right]^{1 / 2}$. The third panel shows the heating efficiency, $\eta=Q_{\text {net }}\left(L_{\nu_{e}}+L_{\overline{\nu_{e}}}\right)^{-1}$. The bottom panel shows the ratio of advection-to-heating time scales.

and $\mathrm{n} 5 \mathrm{~m} 2 f_{\text {heat }} 1.02$ are $0.062,0.080,0.075$, and 0.100 , respectively.

It is almost exactly at the positive inflection in the average shock radius curve of $\mathrm{n} 5 \mathrm{~m} 2 f_{\text {heat }} 1.02(\sim 200 \mathrm{~ms})$ that the critical condition for explosion, $\tau_{\mathrm{adv}} / \tau_{\text {heat }}>1$ is satisfied (Fig. 3; Thompson 2000; Janka 2001; Buras et al. 2006; Fernández 2012). Here we define the average advection time through the gain region as $\tau_{\mathrm{adv}}=M_{\text {gain }} / \dot{M}$ and the gain region heating time as $\tau_{\text {heat }}=\left|E_{\text {gain }}\right| / Q_{\text {net }}$, where $\left|E_{\text {gain }}\right|$ is the total specific energy of the gain region and $Q_{\text {net }}$ is the net neutrino heating in the gain region (c.f. Müller et al. 2012; Ott et al. 2013). During the stalled-shock phase of $\mathrm{n} 5 \mathrm{~m} 2 f_{\text {heat }} 1.02$, around $100-200 \mathrm{~ms}$, the ratio $\tau_{\mathrm{adv}} / \tau_{\text {heat }}$ is growing continuously. Once this critical ratio exceeds unity, thermal energy builds up in the gain region faster than it can be advected out into the cooling layer and the shock begins to expand.

In order to assess the magnitude of the perturbations as they are actually impinging upon the shock, and their effect on the turbulent postbounce flow, we compute the density-weighted radial averages of the Mach number of anisotropic motion,

$$
\left\langle M_{\text {aniso }}\right\rangle=\left\langle\frac{v_{\text {aniso }}}{\left\langle c_{S}\right\rangle_{4 \pi}}\right\rangle_{r},
$$

where the sound speed is first angle-averaged and the velocity 


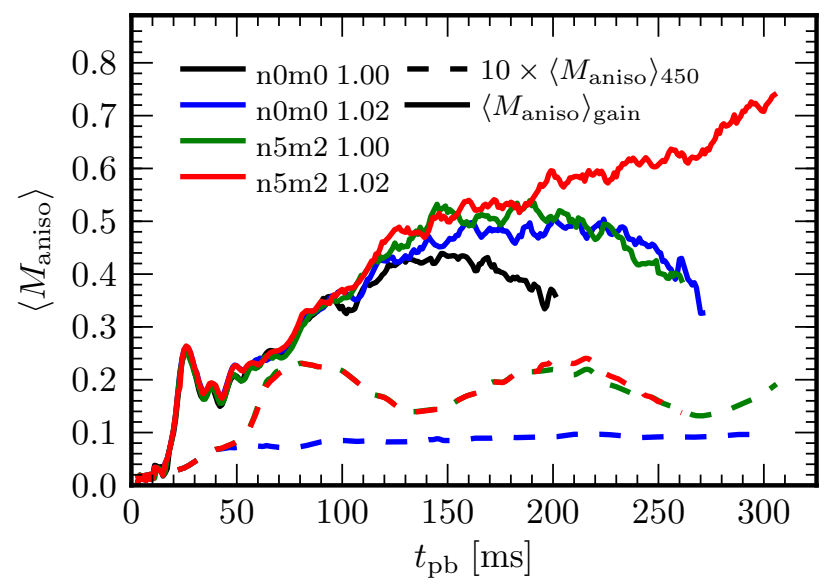

Figure 4. Density-weighted average of the Mach number of anisotropic motion [Eq. (2)] in two separate regions: the gain region (solid lines) and a 100$\mathrm{km}$ wide spherical shell centered on $r=450 \mathrm{~km}$ (dashed lines, multiplied by 10$)$.

of anisotropic motion has the same definition as in Ott et al. (2013); Couch \& O'Connor (2013). The anisotropic Mach numbers for the gain layer and for the preshock radial interval $400-500 \mathrm{~km}$ are shown in Fig. 4. The differences between $\left\langle M_{\text {aniso }}\right\rangle_{450}$ for perturbed and unperturbed cases are evident. The unperturbed cases, n0m0 $f_{\text {heat }} 1.00$ and n0m0 $f_{\text {heat }} 1.02$, have $\left\langle M_{\text {aniso }}\right\rangle_{450} \lesssim 0.01$, whereas in the perturbed models we find peak values of $\left\langle M_{\text {aniso }}\right\rangle_{450}$ of $\gtrsim 0.02$. The Mach number of the perturbations is dramatically reduced by the compression resulting from infall toward the shock. Larger preshock values of $\left\langle M_{\text {aniso }}\right\rangle$ correlate with larger post-shock values of $\left\langle M_{\text {aniso }}\right\rangle$. The perturbed models for both low and high heat factors show similarly large values of $\left\langle M_{\text {aniso }}\right\rangle_{\text {gain }}$ until $\sim 200 \mathrm{~ms}$ when $\mathrm{n} 5 \mathrm{~m} 2 f_{\text {heat }} 1.02$ begins to explode. The unperturbed models have lower values of $\left\langle M_{\text {aniso }}\right\rangle_{\text {gain }}$ than either perturbed model. The Mach number of anisotropic motion for $\mathrm{n} 0 \mathrm{~m} 0 f_{\text {heat }} 1.02$ overtakes that of $\mathrm{n} 5 \mathrm{~m} 2 f_{\text {heat }} 1.00$ around $220 \mathrm{~ms}$, which we attribute to stronger neutrino-driven convection.

Another useful metric of the character of the disturbances reaching the shock is the power spectrum of the perturbations, which we show in Fig. 5. We define the power spectrum of the perturbations to a scalar field $X$ as $\delta_{\ell}^{X}=P_{\ell}^{X \text {,perturbed }}$ $P_{\ell}^{X \text {,unperturbed }}$, where

$$
P_{\ell}^{X}=\sum_{m=-\ell}^{\ell}\left[\oint X(\theta, \phi) Y_{\ell}^{m}(\theta, \phi) d \Omega\right]^{2} .
$$

The spherical harmonics, $Y_{\ell}^{m}$, have their usual definition, and details of similar calculations may be found in, e.g., Hanke et al. (2012); Dolence et al. (2013); Couch (2013). Figure 5 shows the perturbations for tangential velocity, $v_{\tan }=$ $\sqrt{v_{\theta}^{2}+v_{\phi}^{2}}$, and density, where we have set $X$ to the square root of these quantities so $\delta_{\ell}^{X}$ has units of velocity and density, respectively. We normalize $\delta_{\ell}^{v_{\tan }, \rho}$ by the angle-averaged radial velocity and density at $400 \mathrm{~km}$, respectively. The applied perturbations to $v_{\theta}$ manifest themselves in peak values of $\delta_{\ell}^{v_{\tan }, \rho}$ of $\sim 6 \%$ at $\ell=8$. Nonradial motion caused by the initial perturbations results in the growth of density contrasts during infall (Lai \& Goldreich 2000). The peak values of $\delta_{\ell}^{\rho}$ correspond to density contrasts reaching the shock front

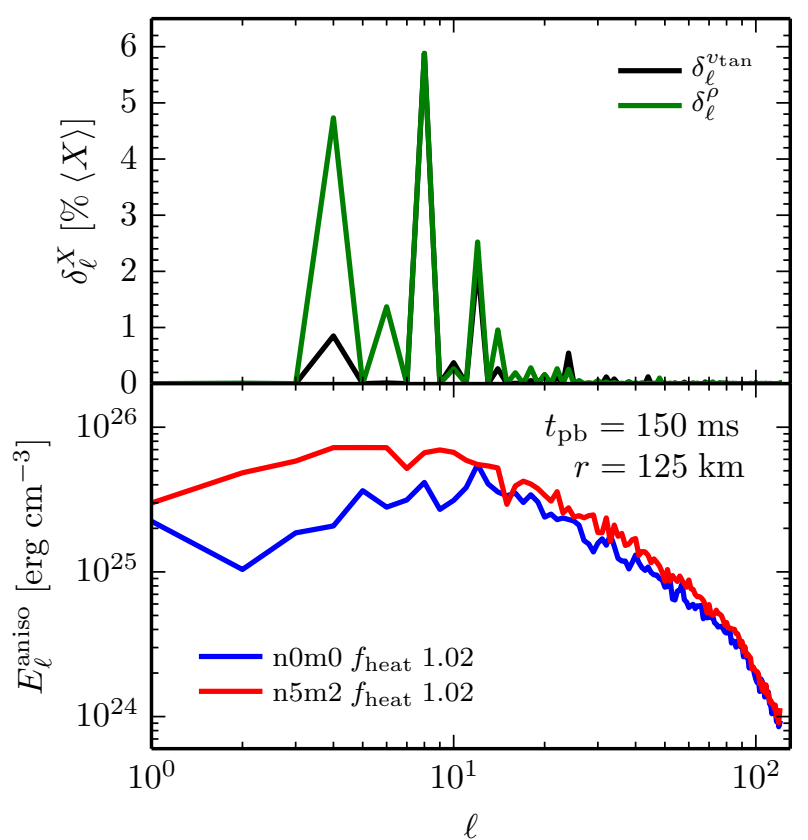

Figure 5. Power spectra in spherical harmonic basis of the perturbations in the pre-shock accretion flow (top). The perturbation spectra are computed as the difference of the sums of the squared spherical harmonic coefficients between the perturbed and unperturbed models, $\mathrm{n} 5 \mathrm{~m} 2 f_{\text {heat }} 1.02$ and $\mathrm{n} 0 \mathrm{~m} 0$ $f_{\text {heat }} 1.00$ [Eq. (3)]. The spectra are computed within a $10 \mathrm{~km}$-wide shell centered on $r=400 \mathrm{~km}$ and averaged over the $10 \mathrm{~ms}$ around $t_{\mathrm{pb}}=100$ $\mathrm{ms}$. Shown are the normalized perturbation spectra of the tangetial velocity (black lines), and of the density field (green lines). We normalize $\delta_{\ell}^{v_{\tan }}$ by the spherically-averaged radial velocity at $r=400 \mathrm{~km}$. The bottom panel shows the anisotropic kinetic energy spectra in the gain region at $t_{\mathrm{pb}}=150$ $\mathrm{ms}$. The spectra are averaged over a $10 \mathrm{~km}$-wide shell centered on $r=125$ $\mathrm{km}$ and averaged over $10 \mathrm{~ms}$. The anisotropic kinetic energy spectra give a measure of the effect of the perturbations on the nonradial flow in the gain region.

of $\geq 2 \times 10^{6} \mathrm{~g} \mathrm{~cm}^{-3}$.

Also shown in Fig. 5 is a powerful diagnostic of the strength of convective and turbulent motions in the gain region, the spectrum of anisotropic kinetic energy, $E_{\ell}^{\text {aniso }}$. It is computed from Eq. (3) with $X=\sqrt{\rho\left[\left(v_{r}-\left\langle v_{r}\right\rangle_{4 \pi}\right)^{2}+v_{\theta}^{2}+v_{\phi}^{2}\right]}$. Model $\mathrm{n} 5 \mathrm{~m} 2 f_{\text {heat }} 1.02$ has significantly more anisotropic kinetic energy at large scales than the unperturbed simulation, n0m0 $f_{\text {heat }} 1.02$. Above $\ell \approx 10$, the spectra of the perturbed and unperturbed cases become fairly similar. The more dramatic difference at small $\ell^{\prime} s$ corresponds to the spatial scales of the perturbations that are reaching the shock, as measured by $\delta_{\ell}$. Kinetic energy on large scales has been noted to correlate with conditions favorable for explosion in a number of previous studies (e.g., Hanke et al. 2012; Couch 2013).

In summary, the message of the various analyses we present in Figs. 3-5 is clear: models with perturbations develop more vigorous postbounce turbulence, have higher neutrino heating efficiencies, and either explode or are much closer to explosion than their unperturbed counterparts. It is particularly noteworthy that the perturbations boost model n $5 \mathrm{~m} 2 f_{\text {heat }} 1.00$ to essentially the same heating efficiency and shock radius evolution as the unperturbed, more strongly heated model n0m0 $f_{\text {heat }} 1.02$. As pointed out by Foglizzo et al. (2006) and first demonstrated by Scheck et al. (2008), the development and strength of neutrino-driven convection in the gain layer increases with increasing magnitude of the accreting seed perturbations. Stronger nonradial motion increases the 
dwell time of material in the gain layer. Thus, our models with perturbations absorb neutrino energy more efficiently, which favors explosion (c.f. Thompson et al. 2005; Murphy \& Burrows 2008).

\section{CONCLUSIONS}

The final phase of nuclear burning in massive stars approaching core collapse is fast and furious. The $\mathrm{Si} / \mathrm{O}$ shells surrounding the iron core are sites of large-scale deviations of turbulent flow from spherical symmetry. Our 3D postbounce CCSN simulations show that aspherical perturbations in the $\mathrm{Si} / \mathrm{O}$ layer can have important effects on the 3D hydrodynamics of CCSNe. They lead to more vigorous turbulent flow behind the shock and qualitatively alter the outcome of core collapse: they can turn a dud into an explosion.

The nonradial momentum-preserving velocity perturbations that we considered here have spatial frequency and Mach numbers comparable to what is expected from 2D Si/O burning simulations (Bazan \& Arnett 1998; Arnett \& Meakin 2011). These perturbations are mild compared to the large $\ell=1$ density variations imposed by the previous studies of Burrows \& Hayes (1996) and Fryer (2004).

Our simulations prove the principle that nonradial velocity perturbations from convective $\mathrm{Si} / \mathrm{O}$ burning can alter postbounce CCSN hydrodynamics and can affect the explosion mechanism. We study the effect of only one particular perturbation, however, it is likely that the outcome will depend on both magnitude and spatial dependence of the perturbations. This must be explored in future work. The 3D oxygen burning simulations of Meakin \& Arnett (2007) suggest that in 3D the Mach numbers of fluctuations may be only half as large as in 2D. However, Meakin \& Arnett (2007) included only the O shell in 3D and Arnett \& Meakin (2011) argue that it is the interplay of $\mathrm{Si}$ and $\mathrm{O}$ burning shells that drives the most violent fluctuations. Thus, we feel that our Mach 0.2 perturbations in the $\mathrm{Si} / \mathrm{O}$ layer in 3D are plausible.

Recent studies comparing 2D and 3D CCSN hydrodynamics suggest that explosions are more readily obtained in $2 \mathrm{D}$ than in 3D (Hanke et al. 2012; Couch \& O'Connor 2013; Couch 2013; Hanke et al. 2013; Takiwaki et al. 2013, but see Dolence et al. 2013 for a differing view). CCSN theory, however, must robustly produce and explain explosions in $3 \mathrm{D}$ to match observations. There are efforts underway by many groups to improve upon current $3 \mathrm{D}$ simulations in treatments of neutrino transport, weak interaction physics, magnetic fields, and gravity with the hope of robustly producing explosions in 3D. Our work shows that the initial conditions also matter, reminding us that the CCSN mechanism is essentially an initial value problem. At least part of the solution to the long-standing supernova problem must lie in multi-D progenitor structure. Full-core, full-3D progenitor evolution simulations to the onset of iron core collapse are urgently needed.

\section{ACKNOWLEDGEMENTS}

We acknowledge helpful discussions with E. Abdikamalov, D. Arnett, P. Goldreich, C. Graziani, C. Meakin, E. O'Connor,
U. C. T. Gamma, C. Reisswig, L. Roberts, and N. Smith. SMC is supported by NASA through Hubble Fellowship grant No. 51286.01 awarded by the Space Telescope Science Institute. CDO is partially supported by NSF grant nos. AST1212170, PHY-1151197, and OCI-0905046 and by the Alfred P. Sloan Foundation.

The software used in this work was in part developed by the DOE NNSA-ASC OASCR Flash Center at the University of Chicago. The simulations were carried out on computational resources at ALCF at ANL, which is supported by the Office of Science of the US Department of Energy under Contract No. DE-AC02-06CH11357, and on the NSF XSEDE network under computer time allocation TG-PHY100033.

\section{REFERENCES}

Arnett, W. D., \& Meakin, C. 2011, ApJ, 733, 78

Bazan, G., \& Arnett, D. 1998, ApJ, 496, 316

Buras, R., Janka, H.-T., Rampp, M., \& Kifonidis, K. 2006, A\&A, 457, 281

Burrows, A., \& Hayes, J. 1996, Phys. Rev. Lett., 76, 352

Chornock, R., Filippenko, A. V., Li, W., et al. 2011, ApJ, 739, 41

Couch, S. M. 2013, ApJ, 775, 35

Couch, S. M., \& O'Connor, E. P. 2013, in preparation

Couch, S. M., Wheeler, J. C., \& Milosavljević, M. 2009, ApJ, 696, 953

Dolence, J. C., Burrows, A., Murphy, J. W., \& Nordhaus, J. 2013, ApJ, 765, 110

Dubey, A., Antypas, K., Ganapathy, M. K., et al. 2009, Parallel Computing, 35,512

Fernández, R. 2012, ApJ, 749, 142

Foglizzo, T., Scheck, L., \& Janka, H.-T. 2006, ApJ, 652, 1436

Fryer, C. L. 2004, ApJ, 601, L175

Fryxell, B., Olson, K., Ricker, P., et al. 2000, ApJS, 131, 273

Hammer, N. J., Janka, H.-T., \& Müller, E. 2010, ApJ, 714, 1371

Hanke, F., Marek, A., Müller, B., \& Janka, H.-T. 2012, ApJ, 755, 138

Hanke, F., Müller, B., Wongwathanarat, A., Marek, A., \& Janka, H.-T. 2013, ApJ, 770, 66

Hobbs, G., Lorimer, D. R., Lyne, A. G., \& Kramer, M. 2005, MNRAS, 360, 974

Janka, H.-T. 2001, A\&A, 368, 527

Joggerst, C. C., Almgren, A., \& Woosley, S. E. 2010, ApJ, 723, 353

Lai, D., \& Goldreich, P. 2000, ApJ, 535, 402

Langer, N. 2012, ARA\&A, 50, 107

Lee, D., Tzeferacos, P., Couch, S. M., \& et al. 2013, in preparation

Meakin, C. A., \& Arnett, D. 2007, ApJ, 667, 448

Müller, B., Janka, H.-T., \& Marek, A. 2012, ApJ, 756, 84

Murphy, J. W., \& Burrows, A. 2008, ApJ, 688, 1159

O'Connor, E., \& Ott, C. D. 2010, CQGra, 27, 114103

Ott, C. D., Burrows, A., Dessart, L., \& Livne, E. 2008, ApJ, 685, 1069

Ott, C. D., Abdikamalov, E., O'Connor, E., et al. 2012, Phys. Rev. D, 86, 24026

Ott, C. D., Abdikamalov, E., Mösta, P., et al. 2013, ApJ, 768, 115

Quataert, E., \& Shiode, J. 2012, MNRAS, 423, L92

Scheck, L., Janka, H.-T., Foglizzo, T., \& Kifonidis, K. 2008, A\&A, 477, 931

Smith, N., \& Arnett, D. 2013, submitted to ApJ, arXiv:1307.5035

Takiwaki, T., Kotake, K., \& Suwa, Y. 2013, Submitted to ApJ, arXiv: 1308.5755

Thompson, C. 2000, ApJ, 534, 915

Thompson, T. A., Quataert, E., \& Burrows, A. 2005, ApJ, 620, 861

Vink, J. 2012, A\&A Rev., 20, 49

Wang, L., \& Wheeler, J. C. 2008, ARA\&A, 46, 433

Woosley, S., \& Heger, A. 2007, Phys. Rep., 442, 269 